\title{
Electromagnetic navigation-guided preoperative localization: the learning curve analysis
}

\author{
Jiang Shi ${ }^{1,2 \#}$, Jiaxi $\mathrm{He}^{1,2 \#}$, Jianxing $\mathrm{He}^{1,2}$, Shuben $\mathrm{Li}^{1,2}$ \\ ${ }^{1}$ Department of Thoracic Surgery, the First Affiliated Hospital of Guangzhou Medical University, Guangzhou, China; ${ }^{2}$ State Key Laboratory and \\ National Clinical Research Center for Respiratory Disease, Guangzhou, China \\ Contributions: (I) Conception and design: J Shi, S Li; (II) Administrative support: S Li, Jianxing He; (III) Provision of study materials or patients: \\ Jiaxi He, S Li; (IV) Collection and assembly of data: J Shi, Jiaxi He; (V) Data analysis and interpretation: J Shi, Jiaxi He; (VI) Manuscript writing: All \\ authors; (VII) Final approval of manuscript: All authors. \\ \#These authors contributed equally to this work. \\ Correspondence to: Jianxing He, MD, PhD; Shuben Li, MD, PhD. Department of Thoracic Surgery, Guangzhou Medical University First Affiliated \\ Hospital, No. 151, Yanjiang Road, Guangzhou 510120, China. Email: hejianxing@hotmail.com; 13500030280@163.com.
}

\begin{abstract}
Background: The electromagnetic navigation bronchoscopy (ENB) was increasingly used to mark small pulmonary nodules (PNs) for video-assisted thoracic surgery (VATS) resection due to high effectiveness and low risk. However, no study reports the learning curve of ENB-guided preoperative localization. In the study, we aimed to describe the learning curve of ENB-guided preoperative PNs localization initially.

Methods: Consecutive PNs cases that underwent ENB localizations between October 2018 and October 2019 by the same surgeon in our center were included in the study. The cumulative sum (CUSUM) method was used to analyze the learning curve of ENB localization.

Results: A total of 89 ENB localization from 64 patients were included in this study. The learning curve was divided into 3 phases: Phase I (the initial 11 cases), Phase II (the 12th to the 47 th cases), and Phase III (the 47 th to the 89 th cases). The success rate of ENB localization has increased with the accumulation of operational experience in 3 phases $(72.73 \%, 91.67 \%$, and $97.62 \%, \mathrm{P}=0.049)$. The distance from the ENB guide wire tip to the center of the lesion in Phase I was significantly longer than those in Phase II and Phase III ( $2.46 \pm 1.76$ vs. $1.36 \pm 0.94$ and $1.47 \pm 0.97 \mathrm{~cm}, \mathrm{P}=0.014$ and 0.027 , respectively). Sex, bronchus sign, and learning curve phase were independent risk factors influencing operative time (OT) $(\mathrm{OR}=8.187,18.847$, and 13.920, respectively).
\end{abstract}

Conclusions: The technical competency, which is indicated by higher success rate, localization accuracy, and shorter OT, for ENB-guided preoperative PNs localization was achieved at the 47th operation.

Keywords: Electromagnetic navigation bronchoscopy (ENB); learning curve; pulmonary nodule localization

Submitted Mar 02, 2021. Accepted for publication Jun 19, 2021.

doi: $10.21037 /$ jtd-21-490

View this article at: https://dx.doi.org/10.21037/jtd-21-490

\section{Introduction}

Lung cancer is one of the most prevailing cancers and the leading cause of cancer-associated death worldwide (1). With the advent of low-dose CT screening, early lung cancer can be detected effectively. This improvement in lung cancer early diagnosis has significantly reduced its mortalities through successfully video-assisted thoracic surgery (VATS) resection in its early stage $(2,3)$. However, the precise localization of some ground-glass opacity (GGO) or semisolid small pulmonary nodules (PNs) is challenging to thoracic surgeons during VATS, especially when the nodules are less than $10 \mathrm{~mm}$ in diameter or when they are more than $10 \mathrm{~mm}$ away from the pleura (4).

Electromagnetic navigation bronchoscopy (ENB) is a navigation technology that converts two-dimensional 


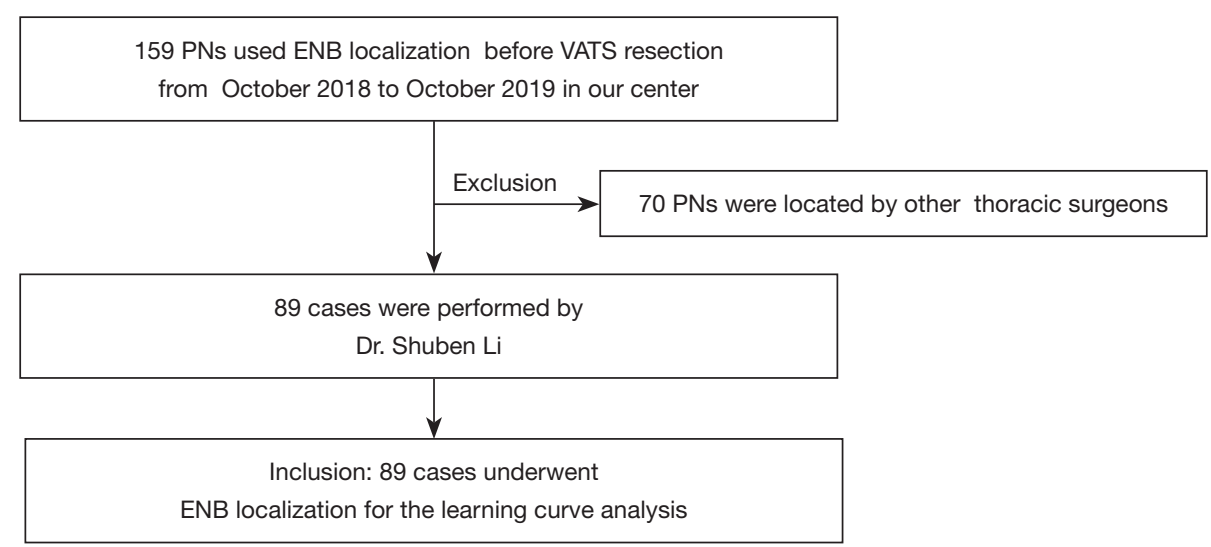

Figure 1 Flow chart of cases selection. PNs, pulmonary nodules; ENB, electromagnetic navigation bronchoscopy; VATS, video-assisted thoracic surgery.

preoperative computed tomographic (CT) imaging into a three-dimensional (3-D) virtual bronchial map and uses an electromagnetic board to guide the bronchoscopic probe to $\mathrm{PN}$ (5). The ENB was used in relatively large PN biopsy initially but with inferior diagnostic yield (6). Recently, this technology was increasingly used to preoperatively mark small PNs due to high effectiveness and low risk $(7,8)$. Many studies have confirmed that ENB was a feasible and effective method for preoperative PN localization with a high success rate $(91.4-97.2 \%)(4,9)$. Compared with the commonly used CT-guided transthoracic localization, the advent of ENB-guided localization can effectively decrease many complications, such as pneumothorax, hemorrhage, and embolism (9). Meanwhile, this technique can be performed in an operating room without radiation exposure. It also has significant advantages in localizing multiple lung nodules compared with the CT-guided method $(10,11)$. However, there was no study to report the learning curve of ENB-guided preoperative localization. In the study, we aimed to initially describe the learning curve of ENB-guided preoperative localization of PNs performed by a single surgeon from our center.

We present the following article in accordance with the STROBE reporting checklist (available at https://dx.doi. org/10.21037/jtd-21-490).

\section{Methods}

\section{Patients and study design}

A total of 129 patients underwent ENB to localize PNs between October 2018 and October 2019 at the First
Affiliated Hospital of Guangzhou Medical University. Among them, 64 patients with 89 ENB localizations, performed by a single thoracic surgeon (SBL) who has the experience of routine bronchoscopy and not the experience of endobronchial ultrasonography (EBUS) and ENB-guided biopsy, were finally included in the study as shown in Figure 1 . The inclusion criteria for ENB localization were: (I) patients with a suspicious malignant appearance in CT scan (GGO or semisolid); (II) nodules between $8-10 \mathrm{~mm}$ in diameter or more than $10 \mathrm{~mm}$ away from the pleura; (III) presumed to be undetectable and unpalpable during VATS based on the surgeon's experience. The cumulative sum (CUSUM) was used to investigate the in-depth learning curve of ENB localization. The study was conducted in accordance with the Declaration of Helsinki (as revised in 2013). The study was approved by Ethics Commission of the First Affiliated Hospital of Guangzhou Medical University (No. 2018-51) and informed consent was taken from all the patients.

\section{ENB localization}

All patients underwent intravenous anesthesia with a laryngeal mask in the operating room. ENB localization was performed as previously reported (12). ENB was performed by using the electromagnetic navigation system (LUNGCARE, Suzhou, China), which plans a visualized route to the target nodules for surgeons based on maximal inspiratory breath-hold (INSP) or expiration (EXP) thoracic CT images. If there was no direct bronchus to the target nodule, we would select the nearest point to the nodule through nearby bronchi. After the locatable electromagnetic guide and the extended working catheter had reached 

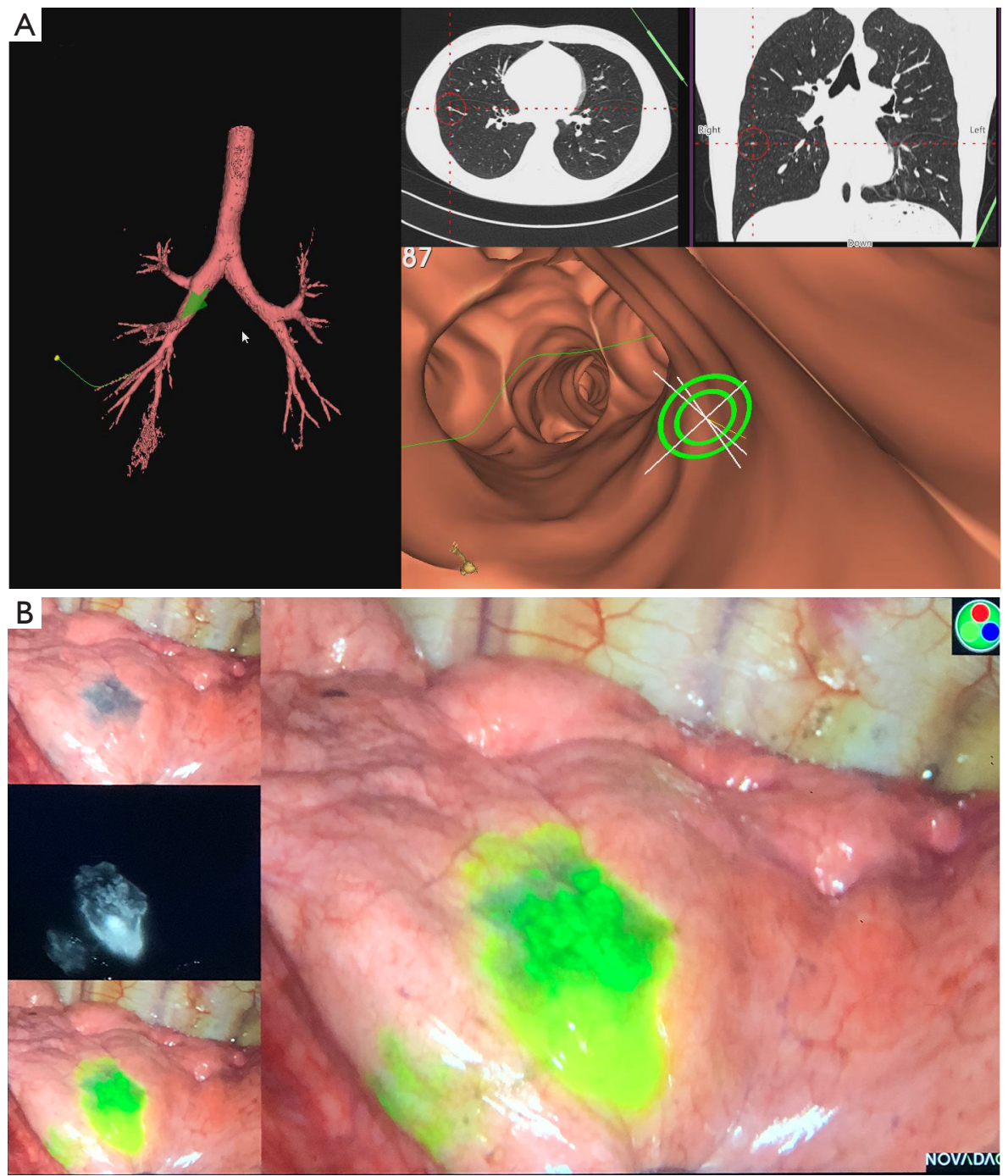

Figure 2 ENB-guided preoperative PN localization. (A) ENB localization system; (B) the ICG marking PN. ENB, electromagnetic navigation bronchoscopy; PN, pulmonary nodule; ICG, indocyanine green.

the target, $0.3 \mathrm{~mL}$ of diluted indocyanine green (ICG) (dilution ration: $1 \mathrm{ml}$ of ICG with $3 \mathrm{~mL}$ of normal saline) or methylene blue and the appropriate amount of air was then injected. The operating process was shown in Figure 2. The patient was then turned to a lateral position and underwent wedge resection by VATS. The successful localization was defined: dye visible during VATS and resection that contained the lesion and dye together.

\section{The cumulative sum method}

In the present study, the cumulative sum (CUSUM) method was used to assess the learning curve, and it determines the accumulated differences among the individual data points and the mean of all data points (13). The patients were categorized in chronological order, and the operative time (OT), spent for the construction of virtual tracheal and the marking of target nodules, was collected for calculating CUSUM CT $_{\left(\operatorname{CUSUM}_{\mathrm{OT}}\right.}=\sum_{i=1}^{n}\left(x_{i}-\mu\right), x_{i}$ and $\mu$ indicates individual OT and the mean OT, respectively). The fluctuation of the learning curve represented different phases of the learning process of the surgeon.

CUSUM was used to analyze marking success as previous report (14). In this study, we determined acceptable failure ratio as 0.1 and unacceptable failure ratio as 0.2 . Conventionally, standard type I error $(\alpha)$ and standard type 
Table 1 General characteristics

\begin{tabular}{|c|c|}
\hline Parameters & Overall \\
\hline \multicolumn{2}{|l|}{ Patients $(n=64)$} \\
\hline Age (years) & $52.58 \pm 12.97$ \\
\hline \multicolumn{2}{|l|}{ Sex, n (\%) } \\
\hline Male & 23 (35.94) \\
\hline Female & $41(64.06)$ \\
\hline \multicolumn{2}{|l|}{ Type of nodule, n (\%) } \\
\hline Solitary nodule & $49(76.56)$ \\
\hline Multiple nodules & $15(23.44)$ \\
\hline \multicolumn{2}{|l|}{ Nodules $(n=89)$} \\
\hline Nodule size $(\mathrm{cm})$ & $0.88 \pm 0.57$ \\
\hline Distance from pleura (cm) & $1.55 \pm 1.13$ \\
\hline \multicolumn{2}{|l|}{ Characteristics, n (\%) } \\
\hline GGO & $69(77.53)$ \\
\hline Solid & $20(22.47)$ \\
\hline \multicolumn{2}{|l|}{ Nodules location, n (\%) } \\
\hline RUL & $19(21.35)$ \\
\hline RML & $6(6.74)$ \\
\hline RLL & $20(22.47)$ \\
\hline LUL & 29 (32.58) \\
\hline LLL & $15(16.85)$ \\
\hline \multicolumn{2}{|l|}{ Bronchus sign, n (\%) } \\
\hline Yes & $18(20.22)$ \\
\hline No & $71(79.78)$ \\
\hline Location time (min) & $11.42 \pm 7.41$ \\
\hline \multicolumn{2}{|l|}{ Pathology, n (\%) } \\
\hline $\mathrm{AAH}$ & $4(4.49)$ \\
\hline AIS & $14(15.73)$ \\
\hline MIA & $40(44.94)$ \\
\hline Invasive adenocarcinoma & $9(10.11)$ \\
\hline Benign & $22(24.72)$ \\
\hline Successful location, n (\%) & $82(92.13)$ \\
\hline
\end{tabular}

RUL, right upper lobe; RML, right middle lobe; RLL, right lower lobe; LUL, left upper lobe; LLL, left lower lobe; AAH, atypical adenomatous hyperplasia; AIS, adenocarcinoma in situ; MIA, minimally invasive adenocarcinoma.
II error ( $\beta$ ) were set as 0.1. CUSUM line below acceptable failure rate boundary (h0) represented the acceptable successful rate of the surgeon.

\section{Statistical analysis}

ENB localization's OT was divided into two groups, 0-14 minutes and more than 14 minutes, according to the mean OT of learning Curve Phase II for multivariable analysis. SPSS 19.0 software was used to perform statistical analysis. Continuous data were expressed as mean \pm standard deviation, and the Student's $t$-test was used to compare the data between the two groups. One-way analysis of variance was used to analyze the data among three groups. Categorical data were analyzed using the chi-square test or Fisher's exact test. Logistic regression models were applied for multivariable analysis. In this study, the $\mathrm{P}$ value of less than 0.05 was considered statistical significance.

\section{Results}

\section{General characteristics}

The general parameters were shown in Table 1. The mean age of the patients was 52.58 years, while females accounted for a larger proportion (64.06\%). The majority of patients (49, 76.56\%) had solitary PNs undergone ENB localization, and 15 patients (23.44\%) had multiple PNs undergone ENB localization. The mean size and length from pleura of PNs were $0.88 \pm 0.57$ and $1.55 \pm 1.13 \mathrm{~cm}$, respectively. The majority of PNs $(69,77.53 \%)$ manifested GGO in CT imaging, and 50 nodules $(56.18 \%)$ located in the upper lobe, followed by in the lower lobe $(31,34.83 \%)$ and the middle lobe $(8$, $8.99 \%)$. Only 18 nodules $(20.22 \%)$ present CT bronchus sign. The mean OT of ENB was $11.42 \pm 7.41$ minutes, and the mean distance between the tip of the locatable guide and the center of the lesion was $1.55 \pm 1.13$ centimeters. The success rate of ENB localization was 82 out of the total 89 cases $(92.13 \%)$. The final pathology revealed $75.28 \%$ of PNs were malignant lung diseases. No complications were found during ENB-guided preoperative localization. In our study, all nodules were resected by VATS without conversion to open thoracotomy. The PNs of unsuccessful localization were performed extend resection or segmentectomy by VATS. 
A

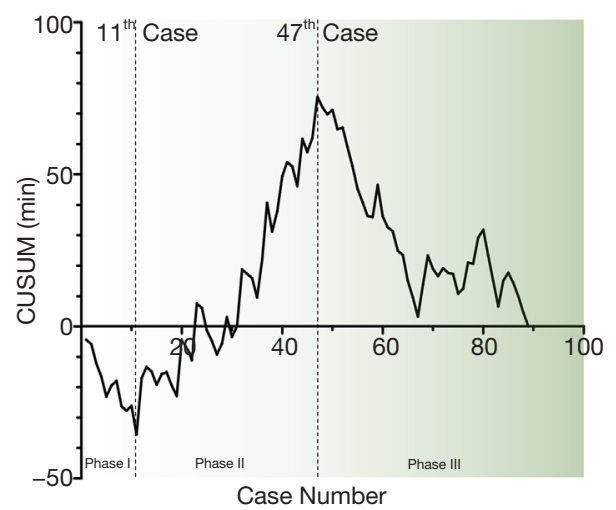

B

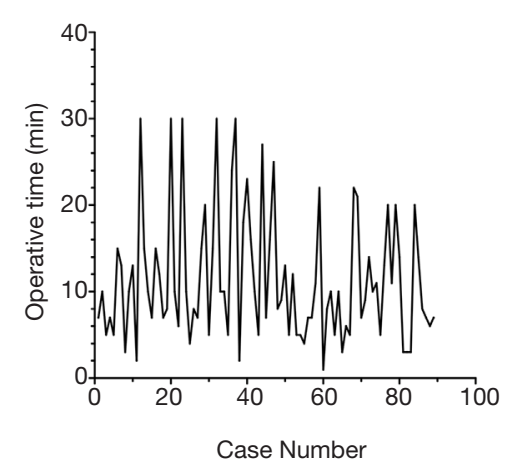

C

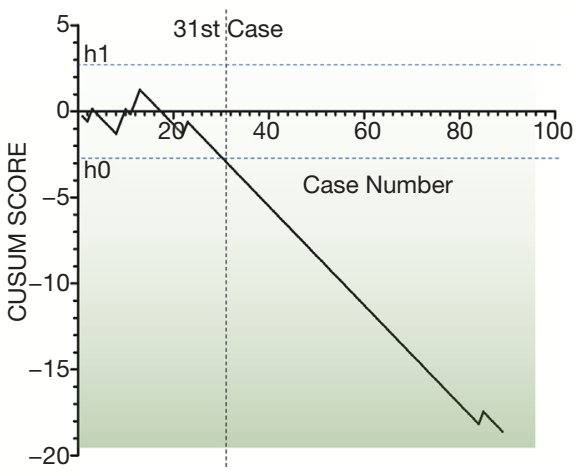

Figure 3 The CUSUM curve of operative time and locating success. (A) The learning curve of ENB-guided PN localization used CUSUM methods for the operative time. As shown in the curve, two cutoff points were found in the $11^{\text {th }}$ case and the $47^{\text {th }}$ case. The learning curve was divided into 3 phases: Phase I (the initial 11 cases), Phase II (the $12^{\text {th }}$ to the $47^{\text {th }}$ case), and Phase III (after the $47^{\text {th }}$ case). (B) The operative time was presented in chronological order. (C) CUSUM of locating success. h1, unacceptable failure rate boundary. h0, acceptable failure rate boundary. The $31^{\text {st }}$ case below h0 represented that the surgeon was competent with an acceptable successful rate. ENB, electromagnetic navigation bronchoscopy; CUSUM, cumulative sum; PN, pulmonary nodule.

\section{Learning curve analysis}

In this study, we used the CUSUM method to analyze the OT of ENB localization in chronological order and drew the learning curve of ENB shown in Figure $3 A$. This figure reveals the change of OT with the increasing experience. And the OT was presented in Figure $3 B$ in chronological order. Two cutoff points were found in the $11^{\text {th }}$ case and the $47^{\text {th }}$ case, respectively. Therefore, the learning curve was divided into 3 phases: Phase I (the initial 11 cases), in which the OT was less than the average OT and the learning curve was in decline trend; Phase II (the $12^{\text {th }}$ to the $47^{\text {th }}$ case), in which the OT was more than the average and the learning curve displayed a continuously rising trend; Phase III (after the $47^{\text {th }}$ case), in which the OT was less than average time and the curve displayed a significantly declining trend. The CUSUM of locating success (Figure 3C) showed that a cutoff point below h0 was made at the $31^{\text {st }}$ case, indicating that the surgeon was competent with an acceptable successful rate.

\section{Nodules characteristics and outcomes of the different learning phases}

The preoperative characteristics and the outcomes of different learning phases were summarized in Table 2. These nodule characteristics, such as type of nodules, nodule size, the proportion of GGO, nodule depth, and final pathology, showed no statistical difference among the three phases. Compared with those in Phase II and Phase III, more PNs were located in the lower lobe in learning Phase I (22.22\%, 33.33\%, and $81.82 \%$, respectively, $\mathrm{P}=0.009)$. The proportion of nodules near a direct airway continuously declined among the 3 phase $(45.45 \%, 25 \%$, and $9.52 \%$, respectively, $\mathrm{P}=0.023)$. As of the outcomes of $\mathrm{ENB}$-guided lung nodules marking, the success rate of ENB localization was increasing with the accumulation of learning experience in 3 phases $(72.73 \%, 91.67 \%$, and $97.62 \%, \mathrm{P}=0.049)$. The distance between marker and lesion in Phase I was significantly longer than in Phase II and Phase III $(2.46 \pm 1.76$ vs. $1.36 \pm 0.94$ and $1.47 \pm 0.97 \mathrm{~cm}, \mathrm{P}=0.014$ and 0.027 , respectively).

\section{Relevant factors influencing $O T$}

The influencing factors determining the operative easinessor-difficulty were shown in Table 3 by univariable analysis and multivariable logistic regression analysis. As shown in univariable, compared with lung nodules with direct airway access, the nodules without a direct airway were more difficult to locate (OT more than 14 minutes) $(\mathrm{P}=0.024)$. The OT longer than 14 minutes had a larger proportion in learning curve Phase II than in Phase I and Phase III $(\mathrm{P}=0.001)$. Though there was no statistical significance about nodule localization, the upper lobe nodules seemed 
Table 2 Preoperative characteristics and outcomes of different learning phase

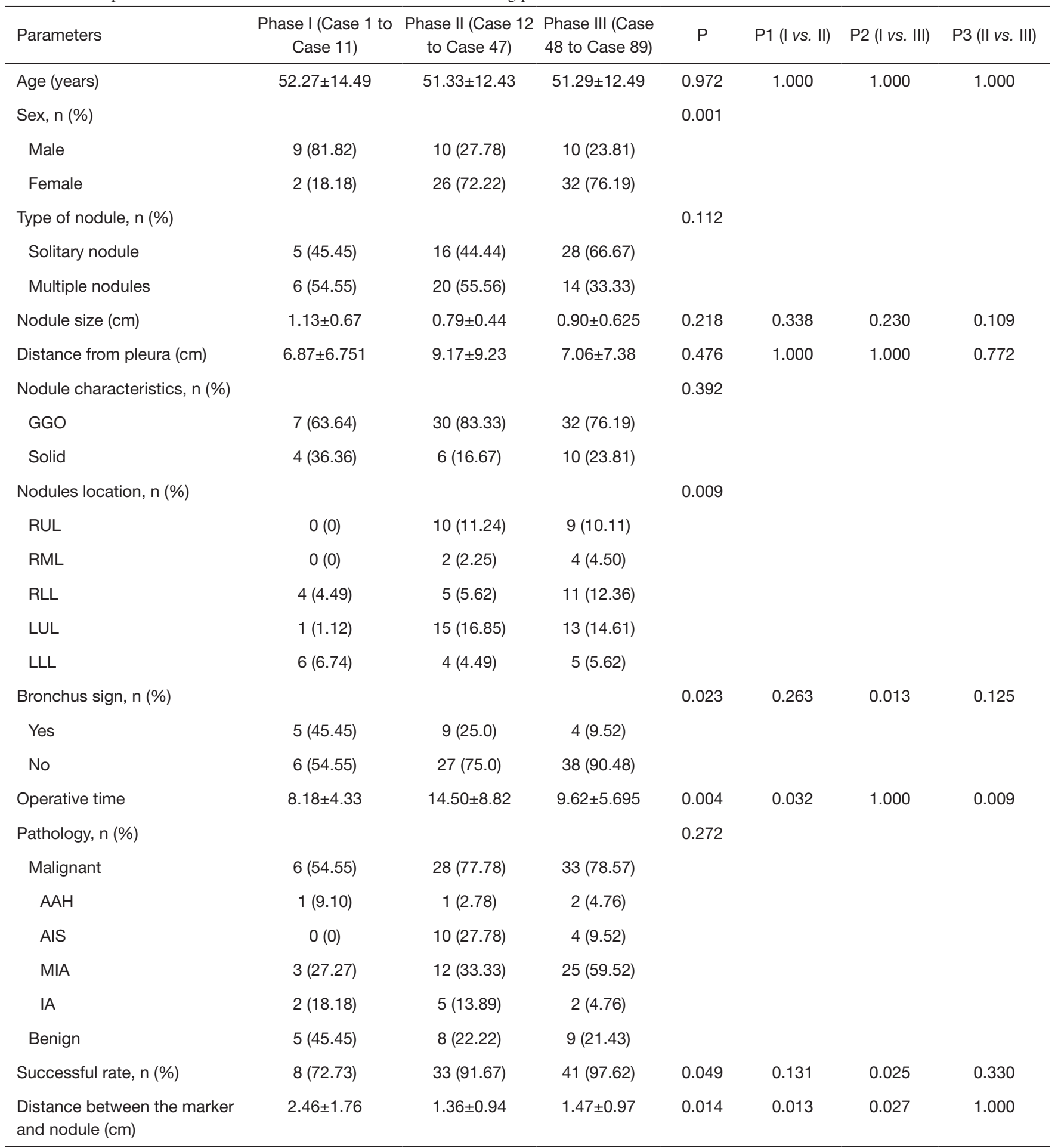

RUL, right upper lobe; RML, right middle lobe; RLL, right lower lobe; LUL, left upper lobe; LLL, left lower lobe. 
Table 3 Relevant factors influences operative time

\begin{tabular}{|c|c|c|c|c|c|c|}
\hline Parameters & $0-14 \min (\mathrm{N}=65)$ & $>14 \min (\mathrm{N}=24)$ & $\frac{\text { Univariable analysis }}{\mathrm{P}}$ & \multicolumn{3}{|c|}{ Multivariable analysis } \\
\hline Age (years) & $51.51 \pm 12.66$ & $51.21 \pm 12.31$ & 0.921 & 0.960 & 1.001 & $0.947-1.058$ \\
\hline Sex, n (\%) & & & 0.105 & 0.015 & & \\
\hline Female & $47(72.31)$ & $13(54.17)$ & & & 1 & \\
\hline Single nodule, n (\%) & $35(53.85)$ & $14(58.33)$ & 0.706 & 0.990 & 1.009 & $0.240-4.252$ \\
\hline Nodule size $(\mathrm{cm})$ & $0.79 \pm 0.44$ & $0.9 \pm 0.53$ & 0.857 & 0.273 & 1.877 & $0.609-5.786$ \\
\hline Distance from pleura $(\mathrm{cm})$ & $14.25 \pm 11.47$ & $18.79 \pm 10.20$ & 0.071 & 0.184 & 0.927 & $0.829-1.037$ \\
\hline Characteristics, n (\%) & & & 0.822 & 0.553 & & \\
\hline Nodules location, n (\%) & & & 0.19 & 0.357 & & \\
\hline Lower lobe & $26(40.00)$ & $5(20.83)$ & & & 1 & \\
\hline Middle lobe & $6(9.23)$ & $2(8.33)$ & & 0.198 & 5.888 & $0.395-87.780$ \\
\hline Upper lobe & $33(50.77)$ & $17(70.83)$ & & 0.210 & 3.095 & $0.530-17.830$ \\
\hline Bronchus sign, $\mathrm{n}(\%)$ & & & 0.016 & 0.024 & & \\
\hline Yes & $17(26.15)$ & $1(4.17)$ & & & 1 & \\
\hline No & $48(73.85)$ & $23(95.83)$ & & & 18.847 & $1.484-239.354$ \\
\hline Phase, n (\%) & & & 0.002 & 0.001 & & \\
\hline
\end{tabular}

to be more difficult to locate than the lower lobe nodules $(17 / 50,34 \%$, vs. $5 / 31,16.13 \%)$. As shown in multivariable logistic regression analysis, sex, bronchus sign, and learning curve phase were independent risk factors of difficult localization. The risk rate of difficult localization was higher in male patients than in female patients ( $\mathrm{OR}=8.187,95 \%$ $\mathrm{CI}=1.504-44.564, \mathrm{P}=0.015)$. Nodules without a presence of bronchus sign had a higher risk for difficult localization than those with bronchus sign (OR $=18.847,95 \%$ CI $=1.484-239.354, \mathrm{P}=0.024)$. The risk rate of OT of more than 14 minutes in the learning curve Phase II was higher than in the learning curve Phase III $(\mathrm{OR}=13.920,95 \%$ CI $=3.182-60.894, \mathrm{P}<0.001)$.

\section{Discussion}

Many previous studies have reported that ENB is useful for localizing the small, non-visible, and non-palpable PNs during VATS. ENB-guided localization significantly decreases complications compared with transthoracic localization with CT-guidance by an interventional radiologist in a radiology suite $(10,15)$. Currently, CTguided preoperative localization is still the most common localization method for small PNs in many thoracic centers. It might partially attribute to the learning curve of ENBguided localization (16). To our knowledge, there was no relative study reporting on the learning curve for ENBguided preoperative localization. 
ENB technology was initially used in the biopsy of relatively large pulmonary lesions. However, there was nonsignificant priority for ENB-guided biopsy compared with CT-guided transthoracic biopsy $(6,17)$. On the contrary, the ENB-guided preoperative localization has significant potentials to mark PNs and assist VATS resection, with a high marking success rate (91.4-97.2\%) nearly without any complication $(4,9)$. Our study showed a similar success rate, especially in the learning curve of Phase III (97.62\%).

In this study, we used CUSUM analysis, commonly used in assessing the trainee competence in new procedures, to detect the differences in ENB OT. When the trainee took more or less time than the mean OT, the learning curve would present rise or fall, respectively (18). In theory, the OT was usually in a gradual decline with the accumulation of learning experiences. And the learning curve usually displays a rising trend in the initial phase and then gradually declines in the expert phase by the CUSUM method reported by previous studies $(18,19)$. However, the learning curve of ENB-guided preoperative localization was a downtrend in Phase I (the initial 11 cases). The ENB-guided localization time was generally less than the mean OT.

To further investigate the influencing factors of OT, we divided the cases into two groups according to location time. We found that the OT of ENB PN localization was affected by the presence of CT bronchus sign and by sex other than by being in different learning phases. The previous reports have confirmed a bronchus sign was associated with highly diagnostic yield for ENB-guided biopsy (20). In this study, we found that the rate of more than 14 minutes in PNs without presence of bronchus sign was higher than that of those with bronchus sign $(\mathrm{OR}=18.847)$. It showed that such factor had more influence on the OT than from the learning curve (OR =13.92, Phases II vs. Phases III). Though there was no statistical significance in nodule localization, the locating time of PNs in the lower lobe was usually less than that in the middle and upper lobes. Most surgeons found it more difficult to complete ENB localization in the upper lobe, because of the vertical angle of the upper lobe bronchus, especially in the apical lung segment (S1). Surprisingly, the male patients spent more locating time than female.

In real clinical situation, it is nearly impossible to avoid selection bias for the early learning curve. And the simple cases would be chosen preferentially when the trainee started to learn a new technique. According to previously reported experiences from ENB-guided biopsy, pulmonary lesions with CT bronchus sign were more likely to be diagnosed compared with the lesions without bronchus sign (20). Therefore, in order to reduce the failure rate of ENBguided localization, the surgeon preferred to select the PNs with bronchus sign or located in the lower lobe in the early learning curve. There was $45.45 \% \mathrm{PNs}$ with bronchus sign and $81.82 \%$ PNs located in the lower lobe in the learning curve Phase I, which were more than Phase II and Phase III. This may be the main reason for the exceptional downtrend of the learning curve Phase I. It may be reasonable to choose simple cases to train ENB-guided preoperative localization not only for operative success rate but for patient's interest. In the study, we confirmed that PNs with bronchus sign or located in the lower lobe were easier to perform ENB-guided localization than PNs without bronchus sign or located in the upper lobe. We recommend choosing PNs with bronchus sign or located in the lower lobe to perform ENB-guided localization preferentially for the new trainees. And we believe the exceptional learning curve of ENB-guided localization reflects the real clinical situation and was suitable to other surgeons.

The success rate of ENB localization and the localization accuracy were both significantly lower in Phase I compared with those in Phase II or Phase III. Apart from the influence of the learning curve, the partly reason for the high failure rate and low accuracy in Phase I could attribute to the imprecise reconstruction of bronchial map, which was compiled based on CT performed at a maximal INSP in advance. Brian S. Furukawa and associates have also reported that PN localization via ENB using EXP CT scans could yield higher accuracy compared with using INSP CT (21). In our study, we used the EXP CT to reconstruct a virtual bronchial map from the $14^{\text {th }}$ Case. It is noteworthy that $0.3-0.5 \mathrm{~mL}$ ICG or methylene blue is enough to mark the PNs. Meanwhile, it is vital for the successful mark to sequentially and slowly administer the right amount of air (3 to 4 milliliters), aimed to push the dye from work catheter to the pulmonary surface of target PNs. In our study, the third case failed to localization without ICG marking caused by the disorderly conduct of administering dye.

Although the OT of the learning curve displayed a generally rising trend with relatively complex cases in Phase II, its ENB localization success rate was close to that of Phase III (91.67\% and $97.62 \%$, respectively), and its marking accuracy was even superior to that of Phase IIII $(1.36 \pm 0.94$ and $1.17 \pm 0.97 \mathrm{~cm}$, respectively). And the CUSUM of locating success indicated that the surgeon was competent with an acceptable successful rate after the $31^{\text {st }}$ case. ICG and methylene blue were both used in our 
study. However, we recommend the ICG dye, which does not hinder observing the lung lesion in VATS resection or jeopardize the further pathological evaluation.

We have to admit there are limitations in the current study. First, it only included cases from a single surgeon and a single ENB operative system. The surgeon with the experience of routine bronchoscopy might affect the learning curve of ENB. More studies from multiple-centers and multiple ENB systems are needed to support our conclusions. Second, the bias of case selections in Phase I may influence the evolution of the learning curve. However, the selection bias may be inevitable for those trainees in clinical practice. Therefore, this unique learning curve represents the reality and is still valuable. Finally, although the ENB performing in the operating room is an effective and low-risk approach to mark small, deep-seated, and non-palpable PNs, it is still more expensive compared with conventional CT-guided methods.

In conclusion, our study demonstrated 3 phases of the learning process of ENB localization before VATS resection. Phase I (the initial 11 easy cases) showed a short operation time but a low success rate and low localization accuracy. Meanwhile, Phase II (12-47 cases) showed a higher success rate and localization accuracy but longer operation time. And the technical competency of high success rate, high localization accuracy, and short operation time was achieved at the $47^{\text {th }}$ operation.

\section{Acknowledgments}

Funding: This study was supported by National Key R\&D Program of China (2017YFC0112700).

\section{Footnote}

Reporting Checklist: The authors have completed the STROBE reporting checklist. Available at https://dx.doi. org/10.21037/jtd-21-490

Data Sharing Statement: Available at https://dx.doi. org/10.21037/jtd-21-490

Peer Review File: Available at https://dx.doi.org/10.21037/ jtd-21-490

Conflicts of Interest: All authors have completed the ICMJE uniform disclosure form (available at https://dx.doi. org/10.21037/jtd-21-490). Jianxing He serves as the
Executive Editor-in-Chief of Fournal of Thoracic Disease. The other authors have no conflicts of interest to declare.

Ethical Statement: The authors are accountable for all aspects of the work in ensuring that questions related to the accuracy or integrity of any part of the work are appropriately investigated and resolved. The study was conducted in accordance with the Declaration of Helsinki (as revised in 2013). The study was approved by Ethics Commission of the First Affiliated Hospital of Guangzhou Medical University (No. 2018-51) and informed consent was taken from all the patients.

Open Access Statement: This is an Open Access article distributed in accordance with the Creative Commons Attribution-NonCommercial-NoDerivs 4.0 International License (CC BY-NC-ND 4.0), which permits the noncommercial replication and distribution of the article with the strict proviso that no changes or edits are made and the original work is properly cited (including links to both the formal publication through the relevant DOI and the license). See: https://creativecommons.org/licenses/by-nc-nd/4.0/.

\section{References}

1. Smeltzer MP, Wynes MW, Lantuejoul S, et al. The International Association for the Study of Lung Cancer Global Survey on Molecular Testing in Lung Cancer. J Thorac Oncol 2020;15:1434-48.

2. Huang W, Deng H, Lan Y, et al. Spontaneous ventilation video-assisted thoracic surgery for mediastinal tumor resection in patients with pulmonary function deficiency. Ann Transl Med 2020;8:1444.

3. Mei J, Guo C, Xia L, et al. Long-term survival outcomes of video-assisted thoracic surgery lobectomy for stage I-II non-small cell lung cancer are more favorable than thoracotomy: a propensity score-matched analysis from a high-volume center in China. Transl Lung Cancer Res 2019;8:155-66.

4. Marino KA, Sullivan JL, Weksler B. Electromagnetic Navigation Bronchoscopy for Identifying Lung Nodules for Thoracoscopic Resection. Ann Thorac Surg 2016;102:454-7.

5. Gildea TR, Mazzone PJ, Karnak D, et al. Electromagnetic navigation diagnostic bronchoscopy: a prospective study. Am J Respir Crit Care Med 2006;174:982-9.

6. Aboudara M, Roller L, Rickman O, et al. Improved diagnostic yield for lung nodules with digital tomosynthesis- 
corrected navigational bronchoscopy: Initial experience with a novel adjunct. Respirology 2020;25:206-13.

7. Awais O, Reidy MR, Mehta K, et al. Electromagnetic Navigation Bronchoscopy-Guided Dye Marking for Thoracoscopic Resection of Pulmonary Nodules. Ann Thorac Surg 2016;102:223-9.

8. Abbas A, Kadakia S, Ambur V, et al. Intraoperative electromagnetic navigational bronchoscopic localization of small, deep, or subsolid pulmonary nodules. J Thorac Cardiovasc Surg 2017;153:1581-90.

9. Hsu PK, Chuang LC, Wu YC. Electromagnetic Navigation-Guided Preoperative Localization of Small Malignant Pulmonary Tumors. Ann Thorac Surg 2020;109:1566-73.

10. Kuo SW, Tseng YF, Dai KY, et al. Electromagnetic Navigation Bronchoscopy Localization Versus Percutaneous CT-Guided Localization for Lung Resection via Video-Assisted Thoracoscopic Surgery: A PropensityMatched Study. J Clin Med 2019;8:379.

11. Tseng YH, Lee YF, Hsieh MS, et al. Preoperative computed tomography-guided dye injection to localize multiple lung nodules for video-assisted thoracoscopic surgery. J Thorac Dis 2016;8:S666-71.

12. He J, Yang H, He J, et al. Electromagnetic navigation bronchoscopy fluorescence localization and VATS subxiphoid bilateral wedge resection under non-intubated anesthesia. J Thorac Dis 2019;11:3186-90.

13. Yap CH, Colson ME, Watters DA. Cumulative sum techniques for surgeons: a brief review. ANZ J Surg 2007;77:583-6.

14. Orhan-Sungur M, Altun D, Özkan-Seyhan T, et al. Learning curve of ultrasound measurement of subglottic diameter for endotracheal tube selection in pediatric

Cite this article as: Shi J, He J, He J, Li S. Electromagnetic navigation-guided preoperative localization: the learning curve analysis. J Thorac Dis 2021;13(7):4339-4348. doi: 10.21037/ jtd-21-490 patients. Paediatr Anaesth 2019;29:1194-200.

15. Lin MW, Tseng YH, Lee YF, et al. Computed tomography-guided patent blue vital dye localization of pulmonary nodules in uniportal thoracoscopy. J Thorac Cardiovasc Surg 2016;152:535-544.e2.

16. Dale CR, Madtes DK, Fan VS, et al. Navigational bronchoscopy with biopsy versus computed tomographyguided biopsy for the diagnosis of a solitary pulmonary nodule: a cost-consequences analysis. J Bronchology Interv Pulmonol 2012;19:294-303.

17. Bhatt KM, Tandon YK, Graham R, et al. Electromagnetic Navigational Bronchoscopy versus CT-guided Percutaneous Sampling of Peripheral Indeterminate Pulmonary Nodules: A Cohort Study. Radiology 2018;286:1052-61.

18. van Zanten F, Schraffordt Koops SE, Pasker-De Jong PCM, et al. Learning curve of robot-assisted laparoscopic sacrocolpo(recto)pexy: a cumulative sum analysis. Am J Obstet Gynecol 2019;221:483.e1-483.e11.

19. Zhang Y, Liu S, Han Y, et al. Robotic Anatomical Segmentectomy: An Analysis of the Learning Curve. Ann Thorac Surg 2019;107:1515-22.

20. Seijo LM, de Torres JP, Lozano MD, et al. Diagnostic yield of electromagnetic navigation bronchoscopy is highly dependent on the presence of a Bronchus sign on CT imaging: results from a prospective study. Chest 2010;138:1316-21.

21. Furukawa BS, Pastis NJ, Tanner NT, et al. Comparing Pulmonary Nodule Location During Electromagnetic Bronchoscopy With Predicted Location on the Basis of Two Virtual Airway Maps at Different Phases of Respiration. Chest 2018;153:181-6. 